Revue d'histoire de l'enfance « irrégulière »

Le Temps de l'histoire

2 | 1999

Cent ans de répressions des violences à enfants

\title{
Le cas des violences scolaires
}

Jean-Charles Basson

\section{(2) OpenEdition}

\section{Journals}

Édition électronique

URL : http://journals.openedition.org/rhei/39

DOI : $10.4000 /$ rhei.39

ISBN : 978-2-7535-1639-7

ISSN : 1777-540X

Éditeur

Presses universitaires de Rennes

Édition imprimée

Date de publication : 15 novembre 1999

Pagination : 193-206

ISSN : 1287-2431

Référence électronique

Jean-Charles Basson, « Le cas des violences scolaires », Revue d'histoire de l'enfance «irrégulière » [En ligne], 2 | 1999, mis en ligne le 30 juillet 2010, consulté le 03 décembre 2020. URL : http:// journals.openedition.org/rhei/39 ; DOI : https://doi.org/10.4000/rhei.39

(c) PUR 


\section{Le cas des violences scolaires}

Jean-Charles Basson $^{(1)}$
Le centenaire de la loi sur la "répression des violences, voies de fait, actes de cruautés et attentats commis envers les enfants" adoptée le 19 avril 1898 est l'occasion, à l'aube de la célébration des 10 ans de l'Institut des hautes études de la sécurité intérieure, d'opérer un retour sur les recherches financées par cet institut sur le thème des violences à enfants. Nous retenons plus particulièrement les violences scolaires qui, outre le fait d'avoir mobilisé neuf équipes de recherche entre 1994 et $1997,{ }^{(2)}$ partagent la particularité avec la loi de 1898 de mettre en scène des enfants victimes ou des enfants coupables.

Une distinction de taille les sépare pourtant : là où l'équation était sans doute commutative, elle est aujourd'hui distributive. En effet, si «le sénateur Bérenger introduit dans la loi de 1898 un amendement qui parle des "enfants victimes ou auteurs" [...], [c'est sans doute qu'à l'époque], derrière l'enfant victime, le magistrat voit facilement un enfant coupable qu'il faut éventuellement punir» (Yvorel, 1997, 29-30). Dans le cas des violences scolaires, il ne s'agit pas de faire de l'enfant victime un enfant coupable qui sommeille, mais de noter que les violences subies par les enfants au sein de l'école sont, bien souvent, des violences administrées par d'autres enfants. Cette distinction n'a toutefois rien de nouveau, puisque sont résumées là les deux missions complémentaires qui sont assignées au système français de protection judiciaire de la jeunesse : la prise en charge des mineurs délinquants et la protection des mineurs en danger moral ou physique. Les uns pouvant être les victimes des autres.

Aborder, sur cette base, la question de la répression des violences scolaires ne s'en trouve pas pour autant facilité. La raison en est simple : aucune des équipes de recherche sollicitées ne mentionne le terme dans ses travaux.
(1) Docteur en science politique. Chargé de recherche à l'Institut des hautes études de la sécurité intérieure (IHESI), Paris.

(2) Les rapports de recherche sont mentionnés en bibliographie, en fin d'article, pp. 205206. Ayant fait l'objet de deux synthèses successives (Emin, Gorgeon, 1996 ; Fabre-Cornali, 1997a), d'une publication récapitulative (Charlot, Emin, 1997) et d'une retranscription des actes d'un colloque (Fabre-Corna1i, 1997b), nous ne citons ici que ces sources, plus accessibles que la littérature grise à laquelle elles renvoient. 
Toutefois une lecture transversale des différentes productions scientifiques permet de dégager deux perspectives : d'une part, le recensement des conditions nécessaires à une répression bien comprise laisse apparaître le paradoxe qu'il y aurait à tenter de réguler la vie scolaire présente par des règles n'ayant plus cours hors de l'école; d'autre part, des alternatives à la répression pure et simple des violences scolaires se font jour qui semblent prendre la mesure de l'inadéquation actuelle de l'école à son environnement direct.

\section{LE PARADOXE DE LA RÉPRESSION DES VIOLENCES SCOLAIRES}

Toute transgression appelle nécessairement une sanction, fut-elle symbolique. Les violences scolaires ne dérogent pas à cette règle de bon sens et de droit élémentaire. Toutefois, la répression de ces dernières ne peut opérer que dans certaines conditions. À la lecture des recherches rassemblées par Bernard Charlot et Jean-Claude Emin, nous en dénombrons au moins six, qui, prises isolément ou regroupées en un système cohérent, attestent clairement de l'impossibilité, dans la situation actuelle, de recourir à un mode de répression raisonné, admis de tous et garant des libertés individuelles. Sans doute qu'au-delà du maintien de l'ordre scolaire, c'est de son inadéquation aux valeurs, aux comportements et aux pratiques de la société toute entière dont il est question. De telle façon que vouloir garantir le premier contre l'évolution de la seconde confine au paradoxe.

\section{Une "vision inflationniste" de la violence scolaire}

La première des conditions nécessaires à une éventuelle répression des violences perpétrées dans le cadre de l'école réside, sans doute, dans l'adoption par l'ensemble des acteurs de la vie scolaire d'une définition commune de ces dernières. Or, victime, selon l'expression de Jean-Pierre Bonafé-Schmitt (Charlot, Emin, 1997, 255), d'une "vision inflationniste de la violence", l'école se voit reprocher de laisser se développer en son sein, tout à la fois, les agressions physiques, le racket ou les différentes formes de vandalisme, et l'ensemble des "incivilités" que sont, par exemple, les 
paroles blessantes, les grossièretés diverses, les bousculades, les interpellations, les humiliations...

Conscients qu'il convient de distinguer les manifestations et registres différents que recouvrent les violences scolaires, Jacques Pain et Emilie Barrier en dressent une typologie dégageant les trois formes les plus couramment recensées : les violences verbales, les violences contre les biens et les violences physiques contre les personnes (Charlot, Emin, 1997, 371373). Les premières englobent l'absentéisme chronique, les retards répétés, le refus des punitions, les inconvenances, les grossièretés, les insolences, les jurons, les injures, les insultes, les crachats, le chantage, le racisme, les menaces de mort... Les violences contre les biens, pour leur part, se décomposent en dégradations des biens des enseignants et des responsables de l'établissement (leur voiture, dans la plupart des cas) et en dégradations de l'institution elle-même : perte ou destruction des cahiers d'appel ou de liaison, graffitis, fumigènes, jets de pierres, bris de vitres, cocktails Molotov, intrusions diverses, effractions, cambriolages, incendies, destructions, vandalisme de toute sorte, sans compter les attaques des voitures de police devant l'établissement. Enfin, les violences physiques contre les personnes peuvent prendre la forme de bousculades, brutalités, jeux violents, bagarres, batailles rangées, menaces avec couteau, embuscades punitives, lynchages, racket, trafic de drogue, viols, port d'arme, harcèlement hors de l'établissement, émeutes...

\section{Une échelle des violences scolaires}

Le tableau apocalyptique de la vie des établissements scolaires français ainsi dressé, reste alors à envisager une hiérarchisation des faits de violence. Se retranchant le plus souvent derrière les catégories retenues par le code pénal («les crimes et delits») d'une part, et les multiples "incivilités" au sens où Sébastian Roché les entend d'autre part, les différentes équipes de recherche vont rarement au-delà. Cécile Carra et François Sicot élaborent pourtant huit catégories de victimation qui contribuent à affiner l'analyse des formes complexes que prennent les violences scolaires. Le manque de respect, la dégradation des affaires personnelles, le vol, le chantage, les coups, le racisme, le racket, les agressions ou harcèlements sexuels dessi- 
nent ainsi la sombre échelle de ces dernières (Fabre-Cornali, 1997a, 112).

De là à en conclure à une sorte de perspective linéaire, déterministe et, au bout du compte, fataliste des exactions commises dans l'enceinte scolaire, il n'y a qu'un pas que Jean-Claude Emin et Catherine Gorgeon $(1996,106)$ franchissent en dégageant "un continuum entre l'élève qui marque son indifférence totale pour ce qu'on lui enseigne, les incivilités [...] souvent quotidiennes et très pénibles à vivre pour les personnels d'enseignement et d'encadrement, le sentiment d'injustice et d'arbitraire fréquemment évoqué par les elèves et, au bout le plus visible de la chaîne, les violences au sens pénal ». Si aucune recherche n'est aussi catégorique dans ses conclusions, la plupart partagent le second trait relevé par les auteurs de la première synthèse : la perception des violences scolaires n'est pas identique pour l'ensemble des parties concernées. C'est pourtant là la troisième condition nécessaire au recours à la répression.

\section{Une perception différenciée de la violence scolaire}

Loïck M. Villerbu, Claude Bouchard et Tifenn Moisan mentionnent utilement que "le rappel des codes [qu'ils nomment également "pratiques de pénalité scolaire"] et/ou la création de nouveaux codes s'appuient essentiellement sur des représentations empiriques des problèmes " (Charlot, Emin, 1997, 220-221). Or, ces représentations ne sont ni communes, ni partagées par l'ensemble des acteurs de l'établissement scolaire. La fréquence, la nature et la portée des faits constatés, subis ou perpétrés sont diversement appréciés selon le groupe de référence de l'auteur et de la victime. Le meilleur exemple est fourni par la recherche de Robert Ballion attestant que "les élèves émettent, pour toutes les transgressions à l'exception du vol, un diagnostic plus sévère que celui des adultes » (Charlot, Emin, 1997, 57-58). Ainsi, si un proviseur sur 100 estime que le racisme et préoccupant dans l'établissement scolaire, 11 élèves sur 100 sont dans le même cas. Les proportions sont respectivement de $13 \%$ contre $19 \%$ pour l'usage de drogue et de $7 \%$ contre $12 \%$ pour l'alcool.

Mais l'écart ainsi constaté n'est pas seulement tributaire du degré de perception des violences scolaires et des représentations qu'elles véhiculent. Il peut également s'expliquer par certaines pratiques routinisées ou stratégiques. Nicole Ramognino, Daniel Frandji, Fabienne Soldini et Pierrette 
Vergès font, par exemple, état d'enseignants qui « semblent ne pas voir ou reconnaître le vol, le racket, l'usage de drogue et même l'envoi de projectile sur leur personne ». De même, certains faits sont systématiquement banalisés : le vol n'est finalement qu'un simple chapardage et la bagarre qu'un "rite de socialisation nécessaire à la construction de l'identité masculine " (Charlot, Emin, 1997, 130-131). Banaliser ou rendre volontairement invisibles les faits de violence scolaire permet alors de nier son incapacité à les résoudre, d'éviter de les affronter et de poser la question du recours à la contrainte, à la répression...

\section{Le "chiffre noir" de la violence scolaire}

Toutefois, prôner une approche phénoménologique de la violence scolaire pose immanquablement la question de la validité de la mesure de cette dernière. C'est la quatrième condition nécessaire à une éventuelle répression de faits et délits qui pour être sanctionnés ou poursuivis doivent être objectivables. Or, Jean-Pierre Bonafé-Schmitt relève que si «la police nationale a élaboré une nouvelle rubrique pour comptabiliser les infractions concernant les faits de violence à l'encontre des élèves et des enseignants [... ], en l'état des données disponibles, [...] il est difficile de se faire une idée assez précise du phénomène [... ], car dans ce domaine il existe aussi un "chiffre noir" en raison aussi bien des modes de constatation (plainte ou mention en main courante) que des qualifications des faits délictueux (infractions pénales ou incivilités), que de la réticence des acteurs et même des victimes à déclarer ce qu'ils ont constaté ou subi. » (Charlot, Emin, 1997, 257-258). ${ }^{(3)}$

\section{La difficulté à dire la loi}

$\mathrm{Au}$ regard des biais et entraves présentés précédemment, on comprend qu'il soit difficile d'envisager, à l'encontre des violences scolaires, un mode répressif susceptible d'être admis et considéré comme légitime par tous les acteurs du monde éducatif. Il faudrait, pour cela, qu'un code, qu'une norme, qu'un contrat, qu'une loi prévalent. Or, cette cinquième condition est également remise en cause.

Angelina Peralva note ainsi d'emblée que les violences scolaires échappent en grande partie "à lidée de transgression légale et même - ce qui est encore
(3) Le bilan 1995 des données statistiques sur la violence à l'école dressé par la Direction centrale de la sécurité publique est repris par Dominique FabreCornali (1997a, 109). 
plus important - à l'idée de transgression normative ». L'auteur y voit la marque de "l'effondrement des bases d'un consensus qui avait permis de travestir ce système très contraignant en un ensemble efficace de mécanismes de discipline douce". C'est le modèle républicain d'intégration par l'école qui trouverait là ses limites, illustrées principalement par la massification et la remise en cause de la légitimité des professionnels opérant en son sein. "Susceptibles d'erreur et peut-être responsables d'injustices graves [dans la vie sociale future de l'élève] ", ces derniers ne seraient plus habilités à dire la loi, à fonder un modèle d'ordre (Charlot, Emin, 1997, 103-105).

Malgré la gravité du jugement, Loïck M. Villerbu, Claude Bouchard et Tifenn Moisan pensent qu'il est possible de remédier à cette situation si on s'applique à éviter « la confusion des registres de pénalité » (Charlot, Emin, 1997, 206-207). Les auteurs en dégagent quatre qu'ils qualifient tantôt de "codes", tantôt de "référents" : le code pénal, le règlement intérieur de l'école, les usages et les coutumes. Ils prêtent à chacun une qualification particulière de l'écart à la norme (respectivement : l'infraction, la désobéissance, l'incivilité et l'irrespect), un traitement sociologique propre (l'éducation civique, la charte, le savoir-faire sur la bienséance, le renvoi aux origines) et des modalités opératoires distinctes : la loi s'impose dans le cadre du code pénal, le règlement se propose à l'élève au sein de son établissement, la bienséance s'explique par les usages et la coutume sacralise la naissance. C'est du respect de chacun de ces registres que dépend la paix scolaire.

Moins catégoriques et partisans d'un modèle simplifié, Jacques Pain et Emilie Barrier adoptent une posture médiane et distinguent deux traitements différents des violences scolaires : le registre pénal et le registre symbolique et moral. Dans le premier cas, le nouveau code pénal " indique deux seuils déterminants dont on n'a, selon eux, guère à discuter : l'atteinte des personnes, physique ou verbale caractérisée; l'atteinte caractérisée des biens » (Charlot, Emin, 1997, 368-369). Recouvrant tout à la fois les brutalités, les brimades, les "incivilités" et le "malmenage", le second registre est moins clairement codifié et fait appel à des notions de respect et de normes sociales et culturelles que l'institution scolaire française, contrairement aux pratiques anglaises ou allemandes, répugne à prendre en compte autrement qu'en tentant de les codifier. Autrement dit, la diffi- 
culté à dire la loi ne réside pas seulement dans la confusion des registres de pénalité, mais également dans la prétention à imposer une juridicisation des relations sociales là où une simple négociation ou une médiation par les pairs suffisent. Tel serait le mal français.

\section{Une communauté scolaire déstabilisée}

Enfin, sixième et dernière condition : pour qu'une éventuelle répression des violences scolaires ait quelque chance d'être perçue comme légitime, il faut encore qu'elle soit assurée par une équipe pédagogique affichant une présence, une cohésion et une mobilisation suffisantes. Les auteurs sont unanimes sur ce point : la qualité et le volontarisme de l'ensemble des membres de l'équipe pédagogique sont les seules véritables garanties contre l'émergence et la banalisation des violences scolaires. "Dans les limites de notre échantillon, écrivent sans ambiguité Eric Debarbieux, Alix Dupuch et Yves Montoya en s'appuyant sur une recherche auprès de 86 établissements scolaires, tous les établissements qui sont en plus grande difficulté que leur donne sociale ne le laissait présager connaissent des conflits d'équipe graves qui sont pour nous plus causes que conséquences de la violence " (Charlot, Emin, 1997, 38). Jacques Pain et Emilie Barrier confirment cette analyse, notant que " l'importance de la distance entre l'équipe de direction et les enseignants est correlée à l'importance attribuée à la violence» (Charlot, Emin, 1997, 378).

Nicole Rainognino, Daniel Frandji, Fabienne Soldini et Pierrette Vergès illustrent les pratiques et les mobilisations que peut envisager une équipe pédagogique motivée autour d'un projet commun par les deux formules suivantes : la "pratique des pieds" et la "pratique des yeux". Il s'agit, dans le premier cas, "d'assurer une présence physique en cours de récréation, aux entrées et aux sorties des enfants qui permet de dénouer les conflits avant même qu'ilséclatent de manière violente » et, dans le second cas, de se livrer à un travail de surveillance (Charlot, Emin, 1997, 131-132).

De même, pour avoir plus d'assurance d'être appliquée et tolérée, la règle scolaire devra encore être reconnue par les élèves et leurs parents. Ce qui sous-entend que, d'une façon ou d'une autre, les premiers participent à la vie de l'établissement et que les seconds y sont associés sous la forme partenariale. En effet, selon Jacques Pain et Emilie Barrier, « indépendamment des 
lieux, lorsque l'organisation de la vie de l'établissement donne une place aux elèves dans son fonctionnement [...] ou bien qu'une écoute est organisée et systématique, que ce soit de façon individuelle ou non, institutionnelle ou informelle, alors la perception de la violence est moins forte " (Charlot, Emin, 1997, 378).

Définies selon des critères peu rigoureux, difficilement objectivables et hiérarchisables, perçues différemment selon le temps, le lieu et les acteurs, mesurées approximativement ou volontairement tues, entretenues par la confusion des règles ou échappant aux codifications normatives, causes et conséquences de la déstabilisation de la communauté scolaire, les violences perpétrées à l'école ne semblent obéir à aucune des conditions élémentaires permettant d'envisager leur répression. Tant et si bien que l'on se trouve devant le paradoxe suivant : minant les bases du modèle traditionnel de régulation de la société scolaire, les exactions commises au sein de l'école se nourrissent, en retour, de son dérèglement généralisé et rendent, de fait, la répression nécessaire, alors même que les modalités justifiant son recours ne sont plus assurées. Par ailleurs, on l'a vu, ces dernières sont soumises à de tels aléas qu'il y a peu de chance qu'elles soient réunies simultanément. Le seraient-elles qu'elles rendraient toute répression inutile et impensable.

Concevoir l'exact contre-modèle du tableau livré précédemment (il verrait les violences scolaires clairement définies et étalonnées, uniformément perçues et vécues, administrativement comptabilisées, répertoriées et traitées par une communauté scolaire soudée et sûre de son bon droit) n'étant ni possible ni, sans doute, souhaitable, il reste à envisager des alternatives à la répression pure et simple des violences scolaires.

\section{LES ALTERNATIVES À LA RÉPRESSION DES VIOLENCES SCOLAIRES}

"La violence dérive en partie de l'incapacité actuelle de l'école à fonder un modèle d'ordre opérationnel et compatible avec l'ensemble des changements historiques dont elle a été l'objet au cours de ces dernières années" (Charlot, Emin, 1997, 113). Le constat sans concession livré par Angelina Peralva en conclusion de sa recherche est, peu ou prou, partagé par l'ensemble des équipes mises à 
contribution, Jean-Paul Payet allant jusqu’à voir dans l'école le dernier espace public de "réparation sociale" où il est possible de "mettre au jour le décalage entre le principe républicain d'intégration et une réalité sociale discriminatoire» (Charlot, Emin, 1997, 153).

Conscients que "l'école "récupère" des conflits qui ne peuvent plus s'exprimer ailleurs » (Fabre-Cornali, 1997a, 116) et que son mode de régulation interne ne peut plus se référer à des principes et à des règles démentis hors de son enceinte, les chercheurs sont unanimes à penser que c'est de la construction d'un nouveau référentiel scolaire dont il est aujourd'hui question. Le chantier est vaste et les priorités sont, sans aucun doute, la mesure du rapport à la norme et la définition du risque encouru à s'en trop détacher. Si le recours systématique au traitement judiciaire des violences scolaires est alors massivement rejeté, trois alternatives distinctes à la répression de ces dernières sont en cours de définition et font l'objet d'appréciations diverses.

\section{Le refus de la juridicisation de la vie scolaire}

"L'byper-réglementation du fonctionnement de l'institution scolaire ", pour reprendre les termes de Jean-Pierre Bonafé-Schmitt, apparait comme la réaction presque instinctive, le corollaire obligé aux violences à l'école. Cette logique juridico-administrative peut se prévaloir de quelques réalisations : création d'une nouvelle infraction sanctionnant "l'intrusion dans les établissements scolaires", renforcement de la coopération éducation-police-justice pour le traitement des faits délictueux, augmentation des personnels de surveillance et de santé, réactivation du règlement intérieur, alternatives au conseil de discipline, élargissement des droits des élèves et de leurs parents contre les décisions scolaires... Mais le pendant de cette tendance consistant à faire "du rappel à la loi le maître mot de la politique de prévention, tout se passe comme si on assistait à une abdication de la part des "éducateurs" au profit des "juristes" pour gérer la conflictualité scolaire» (Charlot, Emin, 1997, 261-262). Les chercheurs ont trop expérimenté les dérives d'une telle division des tâches pour ne pas craindre la généralisation de ce penchant actuel, par ailleurs révélateur du paradigme culturel français qui fait de l'école un lieu d'enseignement à côté duquel interviennent les violences scolaires (Charlot, Emin, 1997, 379-380). 


\section{Les interventions structurelles}

Egalement réactives et semblant ne faire que contenir et différer les difficultés, les interventions de type structurel qui ne sont pas accompagnées d'un mode contractuel de régulation des conflits sont sujettes aux mêmes suspicions. Eric Debarbieux, Alix Dupuch et Yves Montoya recensent pourtant parmi celles-ci celles qui ont notoirement amélioré la situation préoccupante d'un collège du nord de la France : la réduction de la taille des établissements, l'augmentation du nombre des surveillants et le filtrage plus serré des entrées et des sorties (Charlot, Emin, 1997, 40). Percevant bien le danger de voir ces interventions poussées à leur terme, Jean-Claude Emin et Catherine Gorgeon croient utile de rappeler qu'il est "préférable de ne point fermer les établissement vis-à-vis de l'extérieur : il faut plutôt marquer des seuils qu'ériger des barrières, ajoutent-ils " $(1996,112)$. Reste que l'un est plus facile à faire que l'autre.

\section{Le modèle curatif}

Pour d'autres encore, plutôt que réprimer, il convient en quelque sorte de soigner, de traiter le mal. Promoteurs de "diagnostics » préalables au "traitement des insécurités endémiques à l'école ", Loïck M. Villerbu, Claude Bouchard et Tifenn Moisan sont partisans de cette approche. En quête d'" éléments de repérage pour une clinique éducative ", ils préconisent " un diagnostic des positions éducatives en difficulté", "un diagnostic de l'elève en difficulté de vie scolaire " et enfin "un diagnostic d'une vie scolaire en difficulté" " (Charlot, Emin, 1997, 203-206). Egalement à la recherche des pathologies situées aux origines des violences scolaires, il s'agit, pour Daniel Favre et Laurier Fortin, "d'arriver à un sevrage progressif des émotions positives procurées par les comportements violents » (Fabre-Cornali, 1997a, 117). Au-delà du jeu métaphorique qui fait de l'école une institution malade (voire un milieu contaminé et infectieux) au chevet duquel se tiennent les spécialistes, on peut craindre que l'approche fasse de l'élève, à la fois la victime d'une forme nouvelle d'intoxication et l'élément contagieux du système général.

\section{Le modèle managérial}

Enfin, les derniers voient dans la "gestion" des violences scolaires un 
mode pertinent de régulation des conflits, même si elle est le plus souvent retenue faute de mieux et élaborée en situation d'urgence. Angelina Peralva ne le cache pas, qui reconnait que "dès lors [qu'] iln'y a plus d'énonciation possible de la norme et de la transgression en tant que faits sociaux objectifs, [...] l'école se transforme en champ ouvert de conflits, dont la gestion n'est possible qu'à travers une sorte de navigation à vue. [Ce qui contraint les enseignants à élaborer] des stratégies de gestion de la violence [...] qui sont au plus loin d'une définition de type normatif $[. .$.$] : se doter d'une compétence relationnelle (en se faisant connaître et$ reconnaître par des attributs de justice, d'écoute, de capacité de négociation, en étant quelqu'un à qui on peut parler), construire une image de soi (un personnage dont les attributs sont reconnus et définis, non pas sur des principes statutaires, par l'institution, donc par le haut, mais par le bas, dans un cadre de relations sociales), créer certaines routines, établir une certaine ritualisation des comportements, dans le but de les stabiliser » (Charlot, Emin, 1997, 106). On mesure la part d'approximation qui résulte nécessairement de cette construction symbolique et la durée nécessaire à son acceptation pour tous, sachant qu'elle est chaque jour susceptible d'être amendée, contestée ou remise en cause. Mais c'est le propre de la gestion managériale que de tenter d'utiliser au mieux les potentialités et les contraintes inhérentes à une situation donnée...

Dépassant la simple technique de gestion des conflits, Jean-Pierre BonaféSchmitt tente, pour sa part, de minimiser le caractère aléatoire de "l'alternative au modèle disciplinaire " qu'il propose : la médiation scolaire. Soucieux "d'éviter la colonisation du "monde vécu" par le médium du droit ", il préconise de s'appuyer sur une "action orientée vers l'intercompréhension" dans le but d'aboutir à un consensus. Il postule ainsi qu'une "rationalité communicationnelle " peut déboucher d'une "plus grande implication des parties dans le règlement des conflits [...] et d'une compréhension mutuelle de leurs besoins et intérêts " (Charlot, Emin, 1997, 262-263). Il s'agit pour cela de favoriser l'apprentissage de l'écoute mutuelle, de la communication avec les autres, de la gestion de l'agressivité, de la coopération, de la responsabilité, du respect et de la solidarité... Priment alors les notions de contrat, de confiance et d'équité.

Si ces dernières préconisations empruntent, en partie, au mode de régulation scolaire ayant cours jusque-là, comment ne pas voir, en conclusion, 
que le paradoxe qu'implique la mise à jour des conditions nécessaires à une répression des violences scolaires et les alternatives envisagées à ce dernier recours situent l'ensemble des recherches collectées par Bernard Charlot et Jean-Claude Emin en pleine actualité. Nous ne citerons que deux exemples parmi les plus récents.

La synthèse provisoire de la consultation nationale des lycéens lancée en janvier 1998 fait d'ores et déjà apparaitre comme priorité une meilleure qualité des relations humaines fondées sur le respect, l'écoute, la confiance et la disponibilité des enseignants. "Quant à la violence, elle est rarement abordée ouvertement [...], mentionne encore la synthèse. Le plus souvent, elle l'est de manière sous-jacente, sous la forme d'une soumission obligatoire infantilisante mal vécue, avec révolte ou résignation, un manque de considération qui se transforme en découragement, en auto-flagellation ou en bumour noir. » (Le Monde, 12-13 avril 1998).

Par ailleurs, parmi les 135 propositions présentées par la mission interministérielle sur la prévention et le traitement de la délinquance des mineurs, Christine Lazerges et Jean-Pierre Balduyk notent, par exemple, qu'il convient de limiter le recours à l'exclusion des établissements des jeunes les plus difficiles et reprennent un certain nombre d'alternatives à cette extrémité que n'auraient pas reniées les équipes de recherche dont nous avons synthétisé les travaux : instituer des médiateurs scolaires dans chaque académie, ou bien étendre les compétences des brigades des mineurs, qui s'occupent essentiellement des enfants victimes, aux mineurs délinquants... (Le Monde, 17 avril 1998). Autre façon d'envisager le traitement des violences d'enfants à enfants. 


\section{BIBLIOGRAPHIE}

BAILLON R., 1996, La gestion de la transgression à l'école, Rapport de recherche pour le compte du ministère de l'Éducation nationale et de l'Institut des hautes études de la sécurité intérieure, Paris, Centre d'analyse et d'intervention sociologiques.

BONAFE-SCHMITT J.-P., SCHMUTZ N., PAUTONNIER C., 1997, La médiation scolaire, approche comparative France-Etats-Unis, Rapport de recherche pour le compte du ministère de l'Éducation nationale, de l'Institut des hautes études de la sécurité intérieure et de la Délégation interministérielle à la ville, Lyon, Groupe lyonnais de sociologie industrielle.

LES CAHIERS DE LA SECURITE INTERIEURE, 1994, La violence à l'école, 15, ler trimestre.

CARRA C., SICOT F., 1996, Pour un diagnostic local de la violence à l'école : enquête de victimation dans les collèges du département du Doubs, Rapport de recherche pour le compte du ministère de l'Éducation nationale et de l'Institut des hautes études de la sécurité intérieure, Besançon, Laboratoire de sociologie et d'anthropologie, Université de Franche-Comté.

CHARLOT B., EMIN J.-C., (dir.), 1997, Violences à l'école, état des savoirs, Paris, Armand Colin.

COSLIN P.-G., 1997, Les violences an collège, Paris, PUF.

DEBARBIEUX E., 1996, La violence en milieu scolaire, perspectives comparatives portant sur 86 établissements, Rapport de recherche pour le compte du ministère de l'Éducation nationale et de l'Institut des hautes études de la sécurité intérieure, Bordeaux, Département des sciences de l'éducation, Université de Bordeaux III.

EMIN J.-C., GORGEON C., 1996, "Violences à l'école : premiers résultats de la recherche", Cabiers de la sécurité intérieure, 24, 2e trimestre, p. 105-113.

FABRE-CORNALI D., 1997a, "Les violences à l'école", Cabiers de la sécurité intérieure, 29, 3e trimestre, p. 107-119.

FABRE-CORNALI D. (dir.), 1997b, Violence à l'école : état des recherches, Actes du symposium des 26-27 juin 1997, Paris, ministère de l'Éduca- 
tion nationale, de la recherche et de la technologie, Direction de l'évaluation et de la prospective.

FAVRE D., FORTIN L., 1996, Etude des aspects socio-cognitifs de la violence chez les adolescents et développement d'attitudes alternatives utilisant le langage, Rapport de recherche pour le compte du ministère de l'Éducation nationale et de l'Institut des hautes études de la sécurité intérieure, Montpellier, Laboratoire de modélisation de la relation pédagogique, Université de Montpellier III.

PAIN J., 1996, Violences à l'école, étude comparative, Rapport de recherche pour le compte du ministère de l'Éducation nationale et de l'Institut des hautes études de la sécurité intérieure, Nanterre, Université Paris X.

PAIN J., BARRIER E., ROBIN D., 1997, Violences à l'école. Allemagne, Angleterre, France. Une étude comparative européenne de 12 établissements du deuxième degré, Paris, Matrice.

PAYET J.-P., 1997, De l'incident à l'affaire : violences et civilités dans l'école urbaine, Rapport de recherche pour le compte du ministère de l'Éducation nationale et de l'Institut des hautes études de la sécurité intérieure, Lyon, Université de Lyon II.

PERALVA A., 1996, La violence au collège : une étude de cas, Rapport de recherche, Paris, Centre d'analyse et d'intervention sociologiques.

RAMOGNINO N. (dir.), 1997, De la violence en général et des violences en particulier, les violences à l'école : l'étude de trois collèges à Marseille, Rapport de recherche pour le compte du ministère de l'Éducation nationale et de l'Institut des hautes études de la sécurité intérieure, Aix-en-Provence, Laboratoire méditerranéen de sociologie, Université de Provence.

SYR J.-H., 1996, Les violences à l'école : l'exemple de l'académie d'AixMarseille, Rapport de recherche pour le compte du ministère de l'Éducation nationale et de l'Institut des hautes études de la sécurité intérieure, Marseille, Institut de sciences pénales et de criminologie.

YVOREL J.-J., 1997, "L'Enfant, la puissance paternelle et le juge au XIXe siècle”, Cabiers de la sécurité intérieure, 28, 2e trimestre, p 17-31. 